- This article explains what the World Wide Web is and how it works.

- The main features of Microsoft's Internet Explorer 7 web browser are described.

- The concepts of hyperlinks, uniform resource locator (URL) and domain name system (DNS) are introduced.

\title{
PRACTICE
}

\section{Introduction to the World Wide Web}

\author{
P. K. Downes ${ }^{1}$
}

The World Wide Web used to be nicknamed the 'World Wide Wait'. Now, thanks to high speed broadband connections, browsing the web has become a much more enjoyable and productive activity. Computers need to know where web pages are stored on the Internet, in just the same way as we need to know where someone lives in order to post them a letter. This section explains how the World Wide Web works and how web pages can be viewed using a web browser.

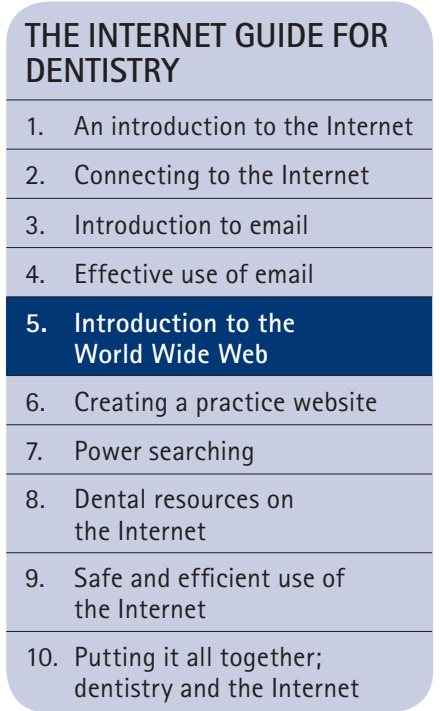

${ }^{1}$ General Dental Practitioner, Kelvin House Dental Practice, 2 Nelson Road, Whitstable, Kent, CT5 1DP

Correspondence to: Dr Paul K. Downes Email:paul@pdownes.fsnet.co.uk

\section{Refereed Paper}

( ) British Dental Journal 2007;

202: $535-540$

DOI: $10.1038 /$ bdj.2007.371

\section{THE WORLD WIDE WEB; WHAT IS IT?}

Although the terms World Wide Web (WWW or simply 'the web') and Internet are often used synonymously, they are actually two different things. Think of the Internet as the structure of interconnected computers and the web as the information and resources that are readily available from these computers.

The web is the world's largest store of easily accessible information, most of it freely available. It is composed of at least 11.5 billion ${ }^{1}$ indexable, interlinked 'pages'. From these pages you can retrieve documents, view images, animation, and video, listen to sound files, speak and hear voice, and run programs.

Who creates all these web pages? Academic establishments, commercial businesses, online stores, media groups, governments and organisations have created many of the web resources available today. However, a large chunk of the web still consists of pages created by individual people who have an interest in a particular topic and who want to make their information freely available to any other interested parties. Think of any obscure topic and I'm sure that you will find that a web page has been written about it!

Web pages are normally grouped together into 'sites'; for example, when people talk about the General Dental Council (GDC) website, they mean the collection of web pages produced by the GDC. Each site is normally, but not always, kept on one computer called a web server. Some web servers will play host to many sites. Because of the ease of navigating from one page to another, it is not normally necessary to know on which computer the information is electronically held.

\section{WHAT MAKES THE WEB SPECIAL?}

The web is quite different from more traditional sources of information such as books, magazines, television and video. There are many reasons for this:

- Web pages can be joined by 'hyperlinks' so that it is easy to jump from one page to another. The link may be between pages on the same site, but it is just as easy to click on a hyperlink and leap to a page on a web server in an entirely different country. A hyperlink normally takes the form of highlighted text (sometimes underlined), or a graphic. As the mouse hovers over a hyperlink, the mouse icon normally turns from an arrow to a pointed finger. An email address appearing on a web page can also be a hyperlink; click on it and your email program will automatically open, create a new message and properly address it 
Fig. 1 The Internet Explorer version 7 toolbar. These icons control the most popular tasks when browsing the web. They are as follows. Navigation icons: (a) back a page, forward a page; (b) go (to the address typed in the address bar); (c) refresh the page, stop downloading the page and the multiple engine search box. Other tasks: (d) home, RSS feeds button (currently greyed-out as the current web page has no feeds), print current page, edit current page, tools for configuration of the browser and help button; (e) favorites and 'add a favorite' buttons (screenshots printed with permission from Microsoft Corporation)
Fig. 2 This screenshot shows Internet Explorer version 7. The address for the General Dental Council (GDC) website has been typed into the address bar. Hitting the Enter key on the keyboard or clicking on the green Go button to the right of the address bar opens up the home index or 'welcome' page for the GDC (Microsoft Internet Explorer screenshot printed with permission from Microsoft Corporation)

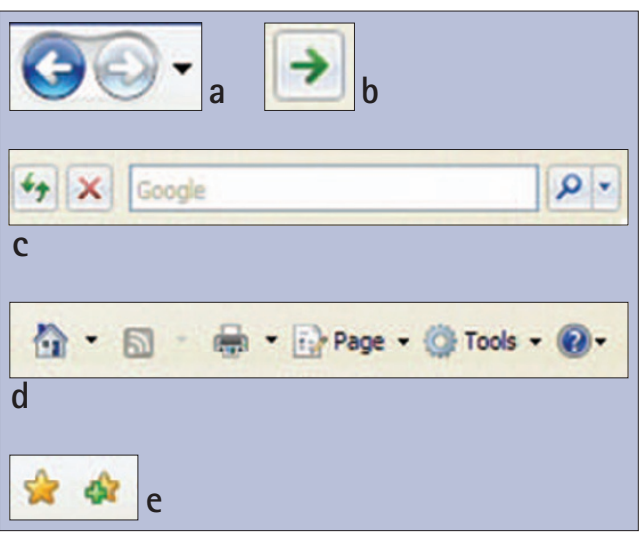

- Because of the way related documents are seamlessly joined by hyperlinks, it can make the process of finding further information on a particular topic almost effortless. For example, you may be looking at a page of information about orthodontics, and then by clicking on some text labelled 'Associations' you may jump straight to a page that contains a list of orthodontic associations with further hyperlinks to their home pages

- It is possible to interact with web pages. Examples include currency converters, ${ }^{2}$ calculators to find the best deal on Internet service, telephone, gas and electricity providers ${ }^{3}$ and interactive forms that allow you to specify multiple details when looking for a second hand car ${ }^{4}$

- The web is becoming a very popular way of buying digital data. Some examples of data being sold on the web include computer programs, updating virus programs, stock digital images for commercial use, ${ }^{5}$ premium content on business sites ${ }^{6}$ and music MP3 files. Apple sold 70 million songs from its iTunes ${ }^{7}$ website at 90 cents a time in its first year of business. It also sold one million videos in less than 20 days when it introduced its video download service in October 2005

- It is easy to update the information held on a web page; for example news sites ${ }^{8}$ and sites that monitor the price of shares ${ }^{9}$ are continually updated

- The web has tools called 'search engines' which enable you to search for a keyword, not just within a particular website, but on billions of web pages. The answer to the search is normally returned within a few seconds

- You can 'publish' your own home/business website and the information it contains is instantly available to anyone with an Internet connection. You would normally upload your created web pages to a web server hosted by your Internet service provider (ISP)

- As we saw in the section on email (BDJ 202: 389-393 and 457-461), the web is increasingly being used as a tool for various forms of communication. Webbased email programs, forums, discussion forums, instant messaging and VoIP telephone calls all help people to communicate information and ideas. One of the latest trends is member communities such as Bebo and MySpace. They represent the next generation of social networking where members can stay in touch with their friends (and friends of their friends), share photos and interests or just 'hang out'. In May 2006, they were the $5^{\text {th }}$ and $6^{\text {th }}$ biggest UK brands online in terms of total web pages viewed.The other big trend is the uploading and sharing of video on sites such as YouTube (bought by Google in October 2006 for \$1.65 billion!).

\section{HOW DOES THE WEB WORK?}

The web is based on a set of rules known as HTTP (hypertext transfer protocol). Web pages can be exchanged and linked to each other by

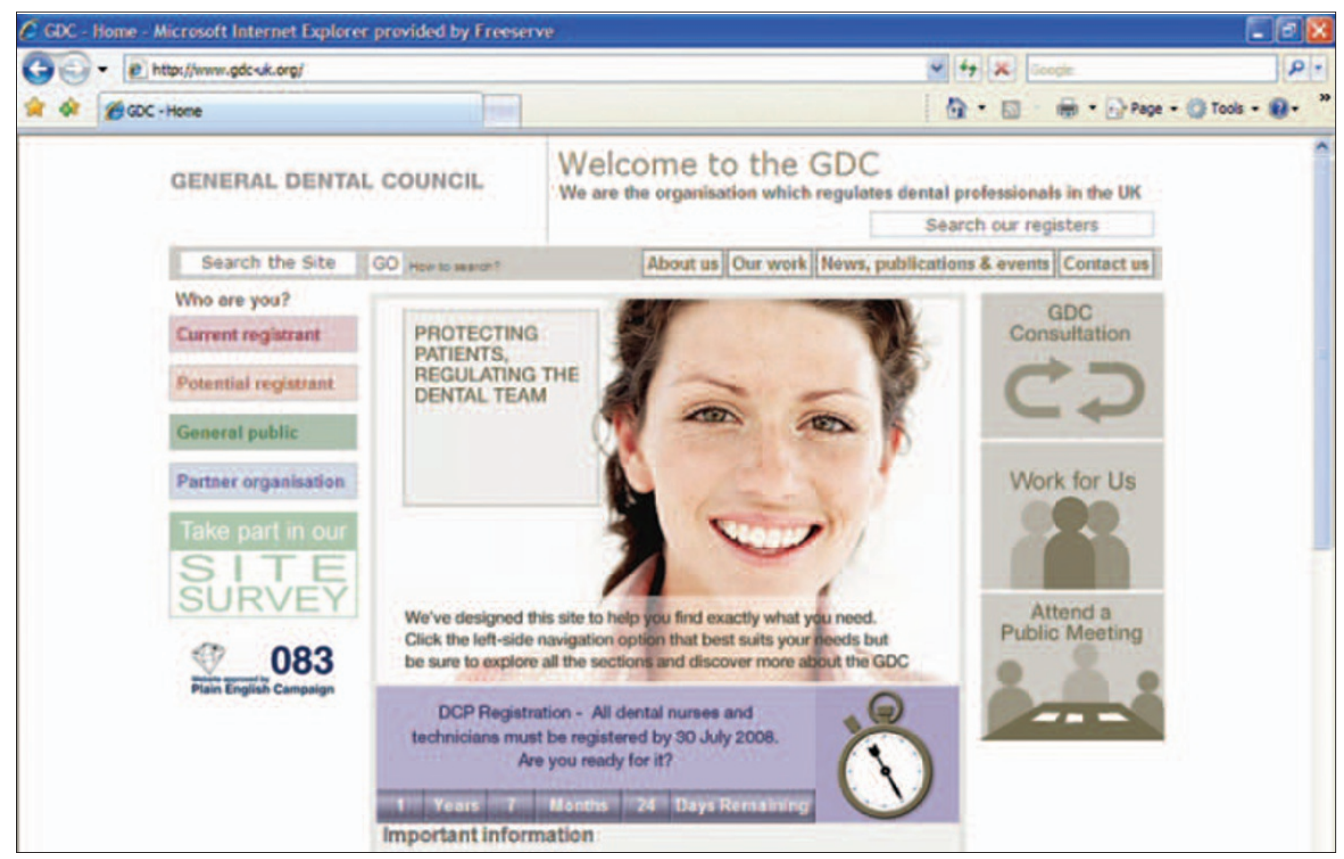


hyperlinks because web browsers (which read the pages) and web servers (which store the pages) both understand HTTP. HTTP defines how information is formatted and transmitted over the Internet and also tells web servers and browsers what action they should take in response to various commands.

For example, when you type a web address into your browser, this actually sends an HTTP command to the necessary web server, directing it to fetch and transmit the requested web page. The other main standard that controls how the web works is HTML (hypertext mark up language), which instructs browsers how web pages are formatted and displayed. It is the programming embedded within HTML that defines any hyperlinks on a web page.

\section{THE WEB BROWSER}

Apart from a computer with an Internet connection, the only other thing you need to 'surf the web' is a software program that enables you to view the web pages. This program is called a web browser, and Internet Explorer (IE) is the most popular web browser, mainly because it is bundled in with the Microsoft Windows operating system. The current version, Internet Explorer 7 (IE7), was publicly released in October 2006 and has better security features than IE6. Other new features include tabbed browsing (making it easier to view numerous web pages), a multiple engine search box (giving greater control over using your favourite search engine as the default), and support for RSS feeds (providing access to frequently updated content on web pages).

IE7 is available as part of Windows Vista, or as a separate download for Windows XP (with Service pack 2) or Windows Server 2003. To see what version you are using, open up IE, click on Help and then click on About Internet Explorer. The latest version can be freely downloaded from the Microsoft website, ${ }^{10}$ which also contains some good tips on how to get the most out of the browser's features. If your computer is running an older Windows operating system, such as Windows 98 or ME, then another option would be to use the freeware plug-in Avant Browser. ${ }^{11}$ This 'sits on top' of IE6 and turns it into a multi-window browser with many extra features.

A guided tour around Internet Explorer version 7 When you start IE it normally opens a default web page; either the home page from Microsoft or from your ISP. If the page is too big to fit on the screen, a scroll bar appears on the right hand side of the page. The most common commands are easily accessible from the large toolbar and address bar (Fig. 1).

There are three main ways of visiting a website:

1. If you know the address for a specific website, open IE and type the web address (see section on 'What is a URL?') into the address bar and hit the Enter key. For

\section{DOMAIN NAMES}

In part 1 we looked at how the Internet uses both a system of names (domain name) and numbers (IP address) to identify computers and individuals on the Internet. Domain names create a single identity for a series of computers used by a company or an institution. So while there may be a dozen or more servers at a given companyl institution, each with its own IP address, they all share a common domain name, such as microsoft.com.

The right-most label in a domain name is referred to as its top level domain (TLD). This three-letter suffix is used to group together certain types of people or institutions. Here are some examples of TLDs:

- $. c o m=a$ commercial organisation

- .org = a non-profit making organisation

- .edu = education

-.gov = government

- .net $=$ network organisations.

Although the majority of .com domain names are registered to US companies, many companies based outside America but with offices and trade relationships worldwide also register a .com domain name. It projects a global image, carries more 'kudos', but remains relatively anonymous in that it doesn't necessarily reveal your whereabouts. When companies use a .com domain, people often assume that it is based in the USA. There are also TLDs with two letters (such as .de, fr, and .uk) that have been established for over 240 countries and external territories and are referred to as 'country-code' TLDs or ccTLDs. Country-specific domains can tell you something more about the person or institution you are dealing with.

The uk country code is separated into sub categories, called second level domains (SLDs). Here are some of the more common SLDs:

- .co.uk for commercial enterprises

- me.uk for personal domains

- org.uk for non-commercial organisations

- Itd and .plc for registered company names only

- .net.uk for Internet service providers

- sch.uk for schools

- .ac.uk for higher education establishments

- gov.uk for government bodies

- .nhs.uk for NHS organisations.

People are encouraged to register a domain name that corresponds to their type of activity. As you can imagine, certain rules and restrictions apply to registering names in some of these categories. ${ }^{15}$

example, if you type in www.gdc-uk.org and either hit Enter or click the green Go button next to the address bar, the browser will open up the home, index or 'welcome' page of the GDC website (Fig. 2)

2. The second way of visiting a website is by following a hyperlink. For example, from the GDC home page, click on the (internal) hyperlink labelled Links; this can be found at the very bottom of the page. This takes you to another page within the GDC website and contains hyperlinks to many 
useful dental websites (Fig. 3). Clicking on one of these external hyperlinks takes you to the home page of the appropriate dental organisation (Fig. 4)

3. A third way of visiting a website is where you do not know the address for the site. This requires carrying out a search of the web using the search engine you have specified in IE (a later section will look at search engines in more detail). Enter your keyword(s) into the search text box and then click on the adjacent Search icon (Fig. 5). A new page is created 'on the fly' which shows the results of your search. Each result is in turn a hyperlink to a web page within a website, but not necessarily the home page.

To return to a previous page, click on the Back button. Use the Forward button to then retrace your steps. If you want to stop a page from downloading, click the Stop button. Sometimes not all the elements of a web page appear first time; if this happens, click the Refresh button. The Home button returns you to the browser's default web page. The Favorites toolbar enables you to store and organise the addresses of your favourite websites.

\section{Other web browsers}

Of course, you do not have to use Internet Explorer as your web browser. There is a range of other browsers that may be more suitable for your particular needs. The best browsers at the time of writing appear to be Mozilla Firefox $^{12}$ and Opera; ${ }^{13}$ both use tabbed windows and are available for free download. Their main advantages over IE7 are that their programs are less 'bloated' and tend to run faster.

\section{COPYRIGHT}

Strictly speaking it is an infringement of copyright to save a web page as a file to your hard disk (let alone print it), unless you have permission from the author. Having said that, your computer will automatically copy the contents of every web page you visit and store it in a 'cache' on your hard disk. The general consensus seems to be that it is not an infringement of copyright for a cache to store web data. This is just as well, since ISPs store huge caches of popular web pages so that they can serve a users request almost instantaneously, without having to continually request the page from the original web server.

Saving and re-using images, sounds and video can be a breach of copyright and you need to check the terms and conditions of each site before doing so. Copyright and the Internet is an ever changing and complex topic but the Copyright Licensing Agency has some useful advice on such issues. ${ }^{14}$

\section{WHAT IS A URL?}

A uniform resource locator (URL) is the Internet's way of indicating the unique address of a particular page on the web so that your browser knows exactly where to go on the server to find a page. The full form for a URL is written as http://www.tesco.co.uk/ or http://www.bbc.co.uk/, but it is more common to use the shortened form, such as www.classicfm.co.uk or www.barclays.co.uk (in everyday speech, URLs are shortened even further into phrases such as channel4. com). These URLs will all take you to the home page or starting point of the company's website; from there you can explore the other pages on the site, for example
Fig. 3 Having clicked on the Links hyperlink at the bottom of the GDC home page, this new web page from the GDC site opens; it shows hyperlinks to various other useful dental websites. Clicking on one of the hyperlinks will open the home page of the appropriate dental organisation. By clicking on a link and holding down the Ctrl keyboard button, the web page will open in a separate tabbed window (see Fig. 4) (Microsoft Internet Explorer screen shot printed with permission from Microsoft Corporation)

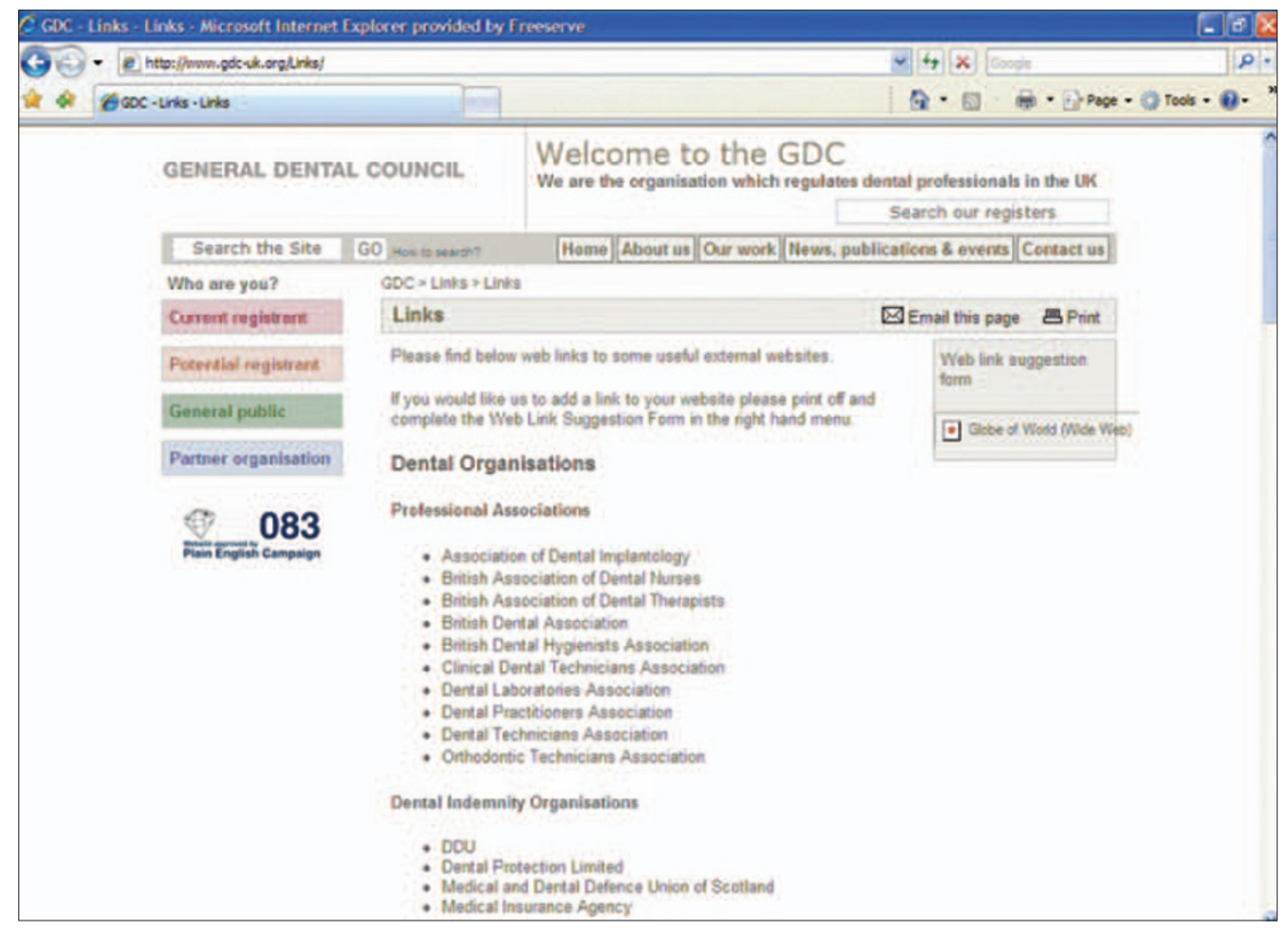




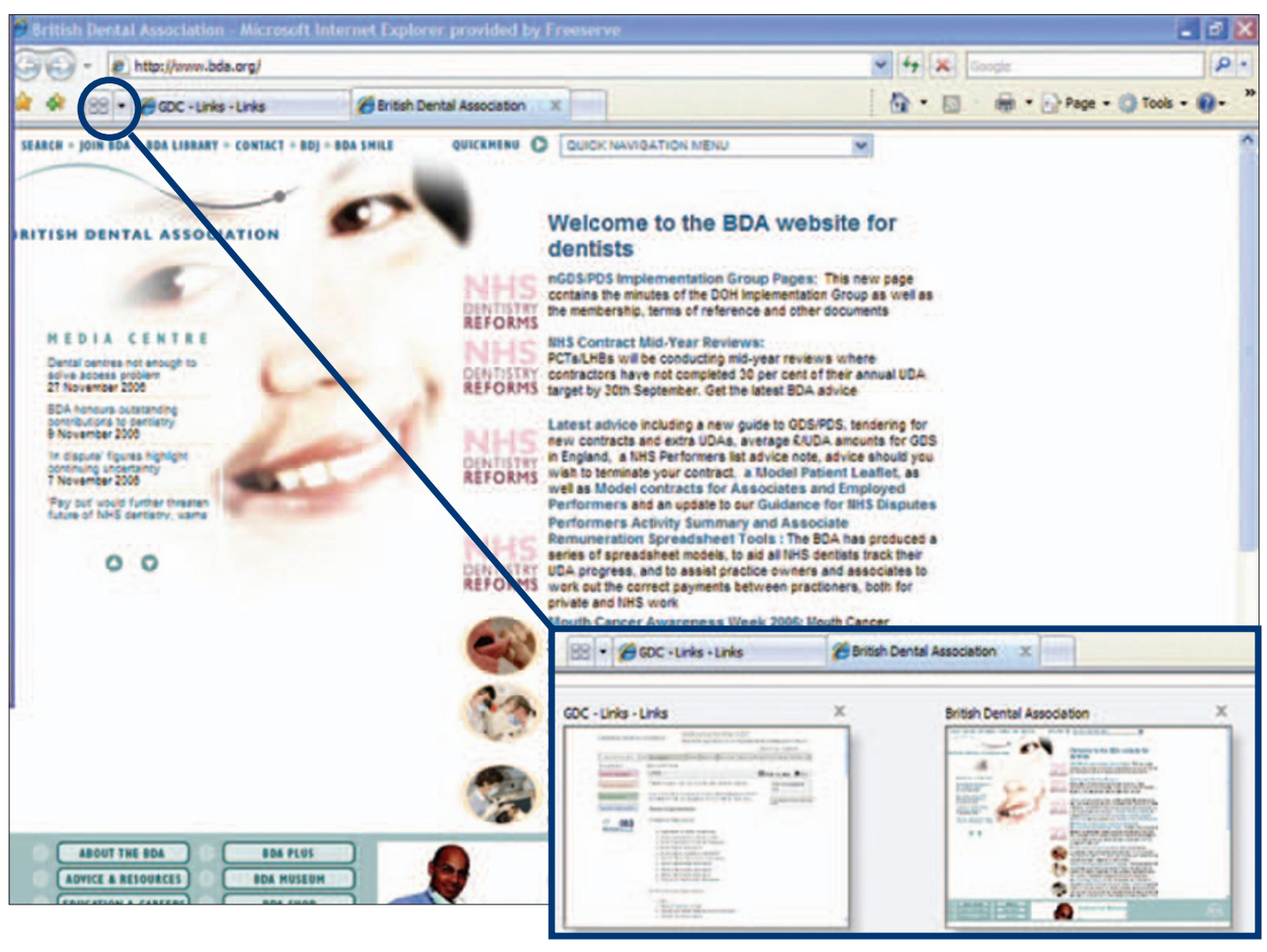

www.movies.co.uk/cinemas/london/plaza. html. This fictitious URL looks a bit like a directory path - which is exactly what it is. It gives the exact location of the web page 'plaza.html' on a particular web server located on the Internet (notice that it uses a forward slash (/) rather than the usual backwards slash ( $\mid$ ) found in the Windows directory structure).

It is not only web pages that have a URL; every document, image, movie or sound file that you find on the web has a unique URL. For example www.ibm.com/sales/map_ europe.jpg would relate to an image map_ europe.jpg. Every hyperlink also has a URL linked to it. When you click on the link, your computer sends a request to retrieve the unique page or item on some computer in the world that is uniquely identified by that URL. Think of URLs as addresses for web pages and the objects that they contain.

\section{WEB BROWSER OR SWISS ARMY KNIFE?}

In the early days of the Internet, there were distinct software programs that you had to use in order to access the different Internet services, for example an email program would just handle email, and you had to use a special newsgroup reader program to access newsgroups and yet another program to

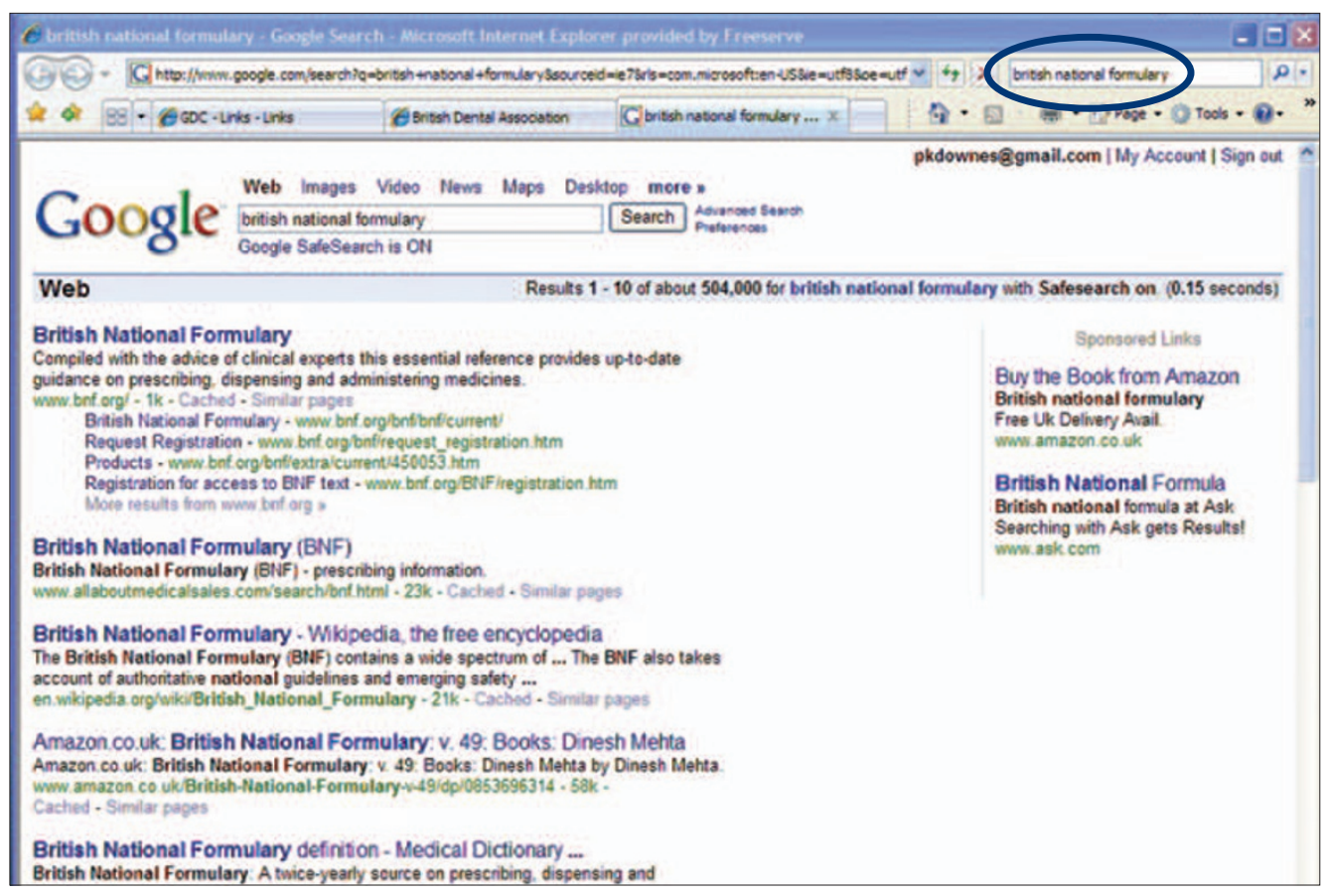

Fig. 4 This screenshot shows the appearance of the British Dental Association website, opened as a second tabbed window. Clicking on the Quick Tabs icon (circled in blue) creates a new page showing thumbnails of all the website windows that are currently tabbed (inset) (Microsoft Internet Explorer screen shots printed with permission from Microsoft Corporation)
Fig. 5 When you do not know the web address for a particular organisation, type suitable keywords into IE7's search text box (circled in blue) to locate their website. In this example we are using Google to search for the terms 'british national formulary'. Click on the Search icon or hit the Enterbutton on the keyboard to see the results page from the search engine. In this instance, the first result is indeed the BNF website, and the text in bold blue is a hyperlink to their home page. Note that in this particular case, by holding down the Alt key at the same time as hitting the Enter key, it has forced the results page to open within a new window. There are now three tabbed windows in the browser (Microsoft Internet Explorer screenshot printed with permission from Microsoft Corporation; Google ${ }^{T M}$ screen capture ${ }^{\odot}$ Google Inc., printed with permission) 
upload files to your website. These standalone programs still exist but the Internet services and the web browser have both evolved so that the web browser of today is the Swiss Army knife of software programs. You can now send and receive web-based email (eg Yahoo! Mail, Hotmail and Gmail), check your normal email account, access newsgroups and mailing lists, upload files to a whole host of different applications (eg online albums of your photographs and website maintenance), and even chat with friends using a web cam. Some of these applications require additional software but the installation and upgrading of such add-on software is so automated that it is easy to forget that it is actually happening.
1. Gulli A, Signorini A. The indexable web is more than 11.5 billion pages. $14^{\text {th }}$ WWW, 2005. http://www.cs.uiowa. edu/ asignori/web-size/

2. OANDA, The Currency Site. http://www.oanda.com/

3. uSwitch. http://www.uswitch.com/

4. Exchange and Mart. http://www.exchangeandmart.co.uk/

5. Corbis commercial images. http://pro.corbis.com/

6. The Economist. http://www.economist.com/

7. Apple iTunes. http://www.apple.com/itunes/

8. BBC News. http://news.bbc.co.uk/

9. Interactive Investor. http://www.iii.co.uk/

10. Microsoft Internet Explorer. http://www.microsoft. com/windows/ie/default.mspx

11. Avant Browser. http://www.avantbrowser.com/

12. Mozilla Firefox 2. http://www.mozilla.org/products/ firefox/

13. Opera web browser. http://www.opera.com/

14. The Copyright Licensing Agency. http://www.cla.co.uk/

15. Nominet: the Internet registry for .uk domain names. http://www.nominet.org.uk/ 